\title{
Nasal Septal Perforation: Experience of Management
}

https://doi.org/10.47210/bjohns.2020.v28i3.296

\author{
Selçuk Kuzu, ${ }^{1}$ Çăglar Günebakan ${ }^{1}$
}

\begin{abstract}
Introduction
ABSTRACT

Nasal septal perforation is the loss of composite tissue comprising the mucosa, bone or cartilage structures that form the nasal septum. Nasal septum perforation has many causes. Though it may be idiopathic, the most common causes are iatrogenic like nasal surgeries. Among other reasons are septal hematoma, nasal picking habit, nasal cauterization due to nosebleeds, nasotracheal intubation, cocaine use, vasculitis, inflammatory diseases such as sarcoidosis, This study aims to review the approach to management of patients with nasal septal perforation who underwent repair of the perforation in a tertiary clinic, in the light of current literature.

Materials and Methods

In this study, the records of 27 patients who were diagnosed with nasal septal perforation and treated surgically in a tertiary clinic, between January 2015 and June 2019 were reviewed retrospectively.

$\underline{\text { Results }}$

The successful closure rate of perforations was $74 \%$. In 4 of 7 patients whose perforations were not completely closed, the perforation size was larger than $2 \mathrm{~cm}$ in diameter.

Conclusion

Successful repair of nasal septal perforation depends largely on the cause, location, size of the perforation, cartilage bone tissue on the perforation edges, surgical technique and the surgeon's experience.

Keywords

Nasal Septum; Nasal Septal Perforation; Surgical Flaps
\end{abstract}

$\mathrm{N}$ asal septal perforation (NSP) is not common and its treatment is difficult. Trauma (nasal surgery, septal fracture, septal hematoma, nasal foreign bodies and nasal piercing, nasal picking, septal cauterization, nasotracheal intubation, etc.), longterm use of nasal spray, smoking habit, cocaine use, inflammation (vasculitis, collagen vascular diseases, sarcoidosis, Wegener granulomatous), infection (tuberculosis, syphilis, lepromatous leprosy, diphtheria, etc.), chemical irritants and neoplasm are among the causes of septal perforation. ${ }^{1-4}$

In recent years, nasal steroid and decongestant sprays are increasingly important causes. Unfortunately, the most common cause of septal perforation are septal surgeries such as submucous resection and septoplasty. ${ }^{5}$ In this study, 27 patients who underwent nasal septal perforation repair in a tertiary clinic were reviewed retrospectively and the approach to nasal septal perforation is presented in the light of current literature.

\section{Materials and Methods}

In this study, the records of 27 patients who were diagnosed with nasal septal perforation and treated surgically in a tertiary clinic, between January 2015 and June 2019 were reviewed retrospectively. Age, gender, size of nasal septal perforation, etiology of the perforation, surgical technique applied, surgical treatment results and period of follow-up were evaluated. According to size

1 - Department of Otorhinolaryngology, Afyonkarahisar

Health Sciences University Medical Faculty, İzmirYolu

Corresponding author:

Dr Selçuk Kuzu

email: dr.selcukkuzu@hotmail.com 


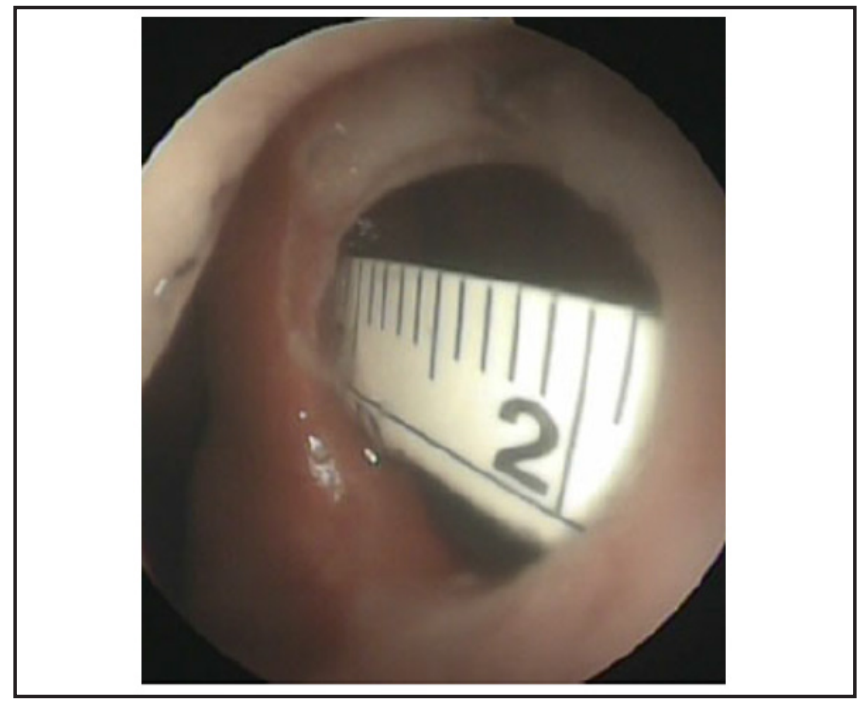

Fig.1. Measurement of an anteriorly placed perforation

of nasal septal perforation; perforations $<0.5 \mathrm{~cm}$ were grouped as small, $0.5-2 \mathrm{~cm}$ as medium and $>2 \mathrm{~cm}$ as large. Perforation repair was performed in all patients with open rhinoplasty under general anesthesia with the help of mucosal flaps and interposition grafts.

Surgical Technique: All cases were operated under general anesthesia. Open rhinoplasty was started with a trans columellar incision. This incision was combined with alar rim incisions on both sides and the back of the nose was elevated. Then, by entering between the medial crura of the alar cartilages, the septum was reached and the upper tunnel was carefully created superior to the perforation. An inferior tunnel was created at the lower edge of the perforation. The mucoperichondrium and then the mucoperiosteum opposite the inferior meatus were elevated on both sides.

After completion of the elevation on both sides, excess septal cartilage or perpendicular laminae of the ethmoid and in some cases, tragal cartilage was harvested for the reconstruction of the perforation. After the edges of the perforation were deepithelialized, four tunnels were created on both sides by the advancement flap technique, and extended up to the top of the nose, the bottom of the nose and the inferior turbinate. Then, longitudinal incisions were made on both sides of the nasal ceiling and mucosa at the base, and the septal flaps were shifted to close the perforation and an interposition

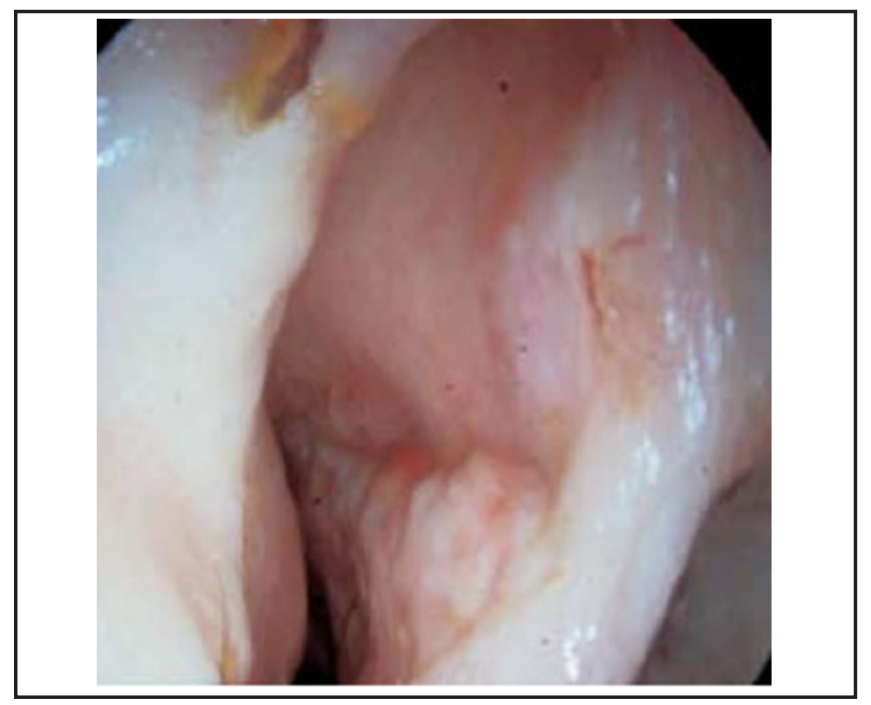

Fig.2. Total closure of perforation,postoperative 5th

graft was inserted. The mucosal flaps were then sutured to the residual mucosa with 5/0 Vicryl ${ }^{\circledR}$ (Ethicon, Inc., Somerville, NJ) in a primer fashion, without being stretched. The dehiscences in the flap donor site at the top and bottom were left for secondary healing. After the operation, internal splints were placed on both sides of the nasal septum after the columellar incision was sutured with a $5 / 0$ prolene suture. Oral broad-spectrum antibiotics and daily saline irrigation were used for 14 days postoperatively. In the postoperative period, patients were warned against smoking, vasoconstrictor sprays, nasal scratching, and straining.

\section{Results}

A total of 27 patients (15 (56\%) males and 12 (44\%) females) were included in the study. The average age of the patient was 32 (24- 55 years old). There was a history of septal surgery in 12 patients (44\%), trauma in 5 patients (19\%) and chronic nasal spray usage in 2 (7\%) patients (Table I).

Etiology could not be determined in $8(30 \%)$ patients. Complaints were nasal obstruction in 10 patients, crusting and bleeding in the nose in 7 patients, only bleeding in 5 patients, and noise in the nose while breathing in 5 patients (Table II). 
Table I: Distribution of patients according to aetiology

\begin{tabular}{|c|c|}
\hline AETIOLOGICAL CAUSE & $\begin{array}{c}\text { NUMBER OF } \\
\text { PATIENTS (\%) }\end{array}$ \\
\hline Previous septal surgery & $12(44 \%)$ \\
\hline Idiopathic & $8(30 \%)$ \\
\hline Trauma & $5(19 \%)$ \\
\hline Nasal Sprey Usage & $2(7 \%)$ \\
\hline
\end{tabular}

Perforation sizes ranged from $0.5 \mathrm{~cm}$ to $3 \mathrm{~cm}$. The size of the nasal septal perforation was small $(<0.5 \mathrm{~cm})$ in $10(37 \%)$ patients, medium $(0.5-2 \mathrm{~cm})$ in $10(37 \%)$ patients, and large $(>2 \mathrm{~cm})$ in $7(26 \%)$ patients. The successful closure rate of perforations was $74 \%$. (Fig. $1,2)$. In 4 of 7 patients whose perforations were not completely closed, the perforation was larger than $2 \mathrm{~cm}$ in diameter (Table III).

The mean postoperative follow-up period of the patients was 4.3 years (10 months- 8 years).

\section{Discussion}

Nasal septal perforation is the loss of composite tissue comprising the mucosa, bone or cartilage that form the nasal septum.6 Nasal septum perforation has many causes. Though it may be idiopathic, the most common causes are nasal surgeries such as submucosal resection, septoplasty, functional endoscopic sinus surgery. ${ }^{7}$ Among other reasons, septal hematoma, nasal picking habit, nasal cauterization for nosebleeds, nasotracheal intubation, cocaine use, vasculitis, inflammatory diseases such as sarcoidosis, Wegener granulomatosis and infections such as tuberculosis, leprosy, syphilis,
Table II: Symptoms in patients with nasal septal perforation distribution

\begin{tabular}{|c|c|}
\hline SYMPTOM & $\begin{array}{c}\text { NUMBER OF } \\
\text { PATIENTS }\end{array}$ \\
\hline Nasal Obstruction & 10 \\
\hline Crusting and bleeding & 7 \\
\hline Bleeding & 5 \\
\hline $\begin{array}{c}\text { Whistling sound from the } \\
\text { nose }\end{array}$ & 5 \\
\hline
\end{tabular}

diphtheria can be listed. ${ }^{8,9}$

The majority of nasal septal perforations are asymptomatic and are diagnosed during examinations done for other reasons. Symptomatic patients have epistaxis, nasal obstruction, discharge, pain and whistling. ${ }^{10}$ Most of the symptoms occur due to turbulence of nasal airflow, which is caused by the perforation. ${ }^{11}$ Posterior perforations cause fewer symptoms than the anterior perforations because the nasal mucosa moistens the respiratory air rapidly. ${ }^{12}$ Symptoms may vary according to location, size, and cause of perforation. A small perforation in the posterior septum can be asymptomatic, but it can cause a distinctly whistling voice when the perforation is anterior. As the size of the perforation increases, laminar air flow deteriorates and turbulence increases. This causes drying, crusting, and nasal congestion. Also, a large anterior perforation may cause saddle nose deformity with loss of structural support. In cocaine users, midto low-grade chondritis may cause pain as in infectious perforations. ${ }^{1,2,13}$ In our study, there were complaints of nasal obstruction in 10 patients, crusting and bleeding in the nose in 7 patients, bleeding in the nose in 5 patients, and noise in the nose in 5 patients.

Table III: Closing rates according to the size of the perforation

\begin{tabular}{|c|c|c|}
\hline PERFORATION SIZE & FULL CLOSURE & NOT CLOSED \\
\hline Small $(<0.5 \mathrm{~cm})$ & 10 & 0 \\
\hline Medium $(0.5-2 \mathrm{~cm})$ & 7 & 3 \\
\hline Large $(>2 \mathrm{~cm})$ & 3 & 7 \\
\hline Total $(27)$ & 20 & 4 \\
\hline
\end{tabular}


Depending on the location and size of the nasal septal perforation, depending on the change in the nasal airflow, patients may have nasal congestion, discharge, crusting, whistling during breathing, nosebleeds, headache, and foreign body sensation in the nose. ${ }^{8}$

Conservative approaches, prosthesis application, and surgery are included in the treatment of nasal septal perforation. Moisturizing and softening ointment applications to the nasal cavity are conservative approaches. The use of septal buttons as a prosthesis is an option. However, ointment application and prosthesis placement approaches are not sufficient to treat symptoms in all patients. The most effective method of restoring normal physiology of the nose is surgical repair. ${ }^{14}$

Many methods of surgical treatment of nasal septal perforation are described in the literature. However, the most important factors affecting the success of septal perforation repair, in addition to the surgeon's ability and experience, are the amount of tissue in the rest of the septum, the size, and location of the septal perforation. ${ }^{7,15}$

The approach is as important as the technique to be preferred in surgery. Closed or open technique can be used for surgical repair of septal perforations. The advantages of the closed technique can be the absence of an external scar, causing minimal tissue damage and minimal damage to anatomical integrity. In contrast to these advantages, an insufficient surgical field of view can be counted as a disadvantage during the suturing of the mucoperichondrial and mucoperiosteal flaps created along with difficulty in placing grafts. The advantages of open technique are a better field of view, easier access to the area of perforation, and the surgeon's ability to use both hands. ${ }^{8}$ The biggest disadvantage of the open technique is that it disrupts the supporting structures and creates an external scar. ${ }^{6}$ In our study, an open approach was applied to all patients.

Techniques commonly described include repair with bilateral septal mucosal flaps with interposition connective tissue grafts, repair with unilateral septal mucosal flaps with or without interposition graft, repair with bilateral septal mucosa flap without nasal graft, nasolabial flap and buccal mucosa flap, composite grafts or flaps. ${ }^{16}$ In our study, perforation repair was performed in patients with the help of advancement mucosal flaps and interposition grafts.

\section{Conclusion}

Successful repair of nasal septal perforation depends primarily on the cause, location, size of the perforation, cartilage and bone tissue at the perforation edges, surgical technique and the surgeon's experience. Since nasal septal perforation is mostly caused by mucoperichondrial and mucoperiosteal tears in nasal surgeries, bilateral mucosal tears should be repaired immediately after detection.

In the light of available literature, it is not possible to pick a single technique that provides guaranteed success in nasal septal perforation repair. The surgical method to be preferred should be determined according to the size, location of the perforation and the amount of tissue left in the remaining septum. To increase the success of surgical results attention should be paid towards creating a contralateral flap prepared from different regions, not stretching the flaps in the repaired area, suture lines not crossing each other and providing a comfortable surgical view. Whichever surgical method is applied, repair of chronic perforation is difficult. For this reason, it would be a more appropriate approach to prevent a septal perforation in the first place.

\section{References}

1. Metzinger SE, Guerra AB. Diagnosing and treating nasal septal perforations. Aesthetic Surg J. 2005; 25:524-9

2. Kridel RW. Septal perforation repair. Otolaryngol Clin North Am. 1999; 32:695-724

3. Rejali SD, Simo R, Saeed AM, de Carpentier J. Acquired immune deficiency syndrome (AIDS) presenting as a nasal septal perforation. Rhinology 1999; 37:93-5

4. Fairbanks DN. Closure of nasal septal perforations. Arch Otolaryngol. 1980;106:509-13

5. Døsen LK, Haye R. Nasal septal perforation 1981-2005: changes in etiology, gender and size. BMC Ear Nose Throat Disord. 2007;7:1

6. Re M, Paolucci L, Romeo R, Mallardi V. Surgical treatment of nasal septal perforations: our experience. Acta Otorhinolaryngol Ital. 2006; 26(2):102-9 
7. Parry JR, Minton TJ, Suryadevara AC, Halliday D. The use of fibrin glue for fixation of acellular human dermal allograft in septal perforation repair. Am J Otolaryngol. 2008; 29: 417-22.

8. Lanier B, Kai G, Marple B, Wall GM. Pathophysiology and progression of nasal septal perforation. Ann Allergy Asthma Immunol. 2007; 99(6):473-9; quiz 480-1, 521

9. Vignes S, Chaillet M, Cabane J, PietteJC. Nasal septal perforation and systemic disease. Rev Med Interne. 2002; 23(11):919-26

10. Brain DJ. Septo-rhinoplasty: the closure of septal perforations. J Laryngol Otol.1980; 94: 495-505

11. Kuriloff DB. Nasal septal perforations and nasal obstruction. Otolaryngol Clin North Am. 1989; 22: 333-50
12. Romo T 3rd, Sclafani AP, Falk AN, Toffel PH. A graduated approach to the repair of nasal septal perforations. PlastReconstr Surg 1999; 103: 66-75

13. Huizing EH, de Groot JA. Septal perforation. In: Huizing EH, de Groot JA, editors. Functional reconstructive nasal surgery. Stuttgart: Georg Thieme Verlag; 2003. p. 180-8

14. Døsen LK, Haye R. Silicone button in nasal septal perforation. Long term observations.Rhinology 2008; 46(4):324-7

15. Tasca I, Compadretti GC. Closure of nasal septal perforation via endonasal approach. Otolaryngol Head Neck Surg. 2006; 135: 922-7

16. Younger R, Blokmanis A. Nasal septal perforations. J Otolaryngol. 1985; 14(2):125. 